\title{
Necesidades de salud en áreas urbanas marginadas de México
}

\author{
Hortensia Reyes-Morales, ${ }^{1}$ Héctor Gómez-Dantés, ${ }^{2}$ \\ Laura del Pilar Torres-Arreola, ${ }^{3}$ Patricia Tomé-Sandoval, ${ }^{4}$ \\ Gloria Galván-Flores, ${ }^{5}$ Marco Aurelio González-Unzaga ${ }^{5}$ \\ y Gonzalo Gutiérrez-Trujillo ${ }^{6}$
}

Forma de citar

Reyes-Morales H, Gómez-Dantés H, Torres-Arreola LP, Tomé-Sandoval P, Galván-Flores G, GonzálezUnzaga MA, et al. Necesidades de salud en áreas urbanas marginadas de México. Rev Panam Salud Publica. 2009;25(4):328-36.

RESUMEN Objetivo. Evaluar las necesidades de salud de la población residente en áreas urbanas marginadas de México.

Métodos. Encuesta poblacional a familias residentes en colonias urbanas pobres de cinco regiones geográficas de México (Norte, Centro, Sur, Sureste y Ciudad de México), seleccionadas mediante un muestreo polietápico. Se realizaron entrevistas y mediciones antropométricas en sus domicilios a todos los integrantes de las familias seleccionadas y se analizaron las características de salud positiva, nutrición, salud reproductiva, daños a la salud y salud mental por grupos de análisis formados por edad y sexo.

Resultados. Participaron 24707 personas. En los entrevistados se observó una baja escolaridad (6 años o menos) y solo $46,8 \%$ tuvo cobertura de servicios de salud, ya fuera en instituciones públicas o privadas. De los niños, $19,8 \%$ presentaba desmedro y el sobrepeso predominó a partir de la adolescencia. En los adolescentes de 12 a 19 años, 15,7\% tenía vida sexual activa, pero solo $57,7 \%$ de los hombres y $41,9 \%$ de las mujeres de ese grupo de edad usaban algún método anticonceptivo. De los adultos, 5,9\% padecía diabetes y 11,5\% hipertensión arterial. En la muestra de adolescentes, adultos y adultos mayores, el tabaquismo fue de 21,2\%, el consumo de alcohol de 36,0\%, el uso de drogas de 9,5\% y la depresión de 20,2\%.

Conclusiones. La vulnerabilidad sanitaria de las personas que viven en las áreas urbanas marginadas de México se manifiesta en la existencia de una población joven, con pocas redes de apoyo familiar y de servicios de salud, que sufre trastornos y enfermedades, como la desnutrición infantil, el alto riesgo reproductivo y las adicciones en adolescentes y adultos, producto del rezago en el desarrollo social. La prevalencia de enfermedades crónicas fue similar a la de la población mexicana no marginada.

Palabras clave Áreas de pobreza, evaluación de necesidades, estado de salud, México.

1 Centro de Investigación en Sistemas de Salud, Instituto Nacional de Salud Pública, Cuernavaca, Morelos, México. La correspondencia se debe dirigir a Hortensia Reyes-Morales, Centro de Investigación en Sistemas de Salud, Av. Universidad No. 655, Col. Santa María Ahuacatitlán, Cuernavaca, Morelos 62508, México. Correo electrónico: hreyes @insp.mx

2 Observatorio de la Salud, Fundación Mexicana para la Salud, México, D.F., México.

3 Coordinación de Unidades Médicas de Alta Especialidad, Instituto Mexicano del Seguro Social, México, D.F., México.
El crecimiento poblacional de las ciudades es un fenómeno mundial y progresivo. Según datos de la Organización

\footnotetext{
4 Coordinación de Investigación en Salud, Instituto Mexicano del Seguro Social, México, D.F., México.

5 Unidad de Investigación Epidemiológica y en Servicios de Salud, Centro Médico Nacional Siglo XXI, México, D.F., México.

6 Hospital Infantil Federico Gómez, México, D.F., México.
}

de las Naciones Unidas (ONU), 49\% de la población mundial residía en ciudades en 2005. Durante el siglo pasado, la urbanización fue resultado del desarrollo económico provocado por la industrialización. Este fenómeno continúa en los países en desarrollo, lo que se refleja en el número creciente de personas que actualmente radica en ciudades de países en desarrollo, cifra que será cuatro veces 
mayor en 2030. Esto significa que globalmente, el crecimiento poblacional se ha convertido en un fenómeno urbano ligado a las regiones menos desarrolladas (1).

Uno de los mayores retos de estos cambios demográficos sin precedentes es el de la atención a la salud de los grupos asentados de manera irregular en la periferia de los centros urbanos. Además de los riesgos y las condiciones de salud, que son específicos debido al ambiente físico de los grandes conglomerados, los aspectos sociales son fundamentales para determinar las necesidades de salud de la población (2). Las áreas de extrema pobreza en las ciudades han surgido como un fenómeno derivado de la inequidad social; en estas áreas se concentran tanto la población marginada de las propias ciudades como la que migra de las áreas rurales por la falta de empleo y posibilidades de desarrollo (3). Estas poblaciones viven en condiciones de estrés social, manifestado por la deprivación económica, el hacinamiento, la inestabilidad familiar, la insalubridad de la vivienda y el ambiente y la inseguridad, condiciones que de manera aislada y en conjunto influyen en la mortalidad prematura $(4,5)$. Asimismo, algunos estudios han demostrado que tanto las condiciones de las viviendas como las de los propios vecindarios influyen en la salud de sus habitantes $(6,7)$. Por otro lado, las condiciones de marginalidad y pobreza exponen a estos grupos a un espectro muy amplio de riesgos para su salud, que no son identificados ni atendidos debido a su falta de acceso a los servicios de salud (8).

Uno de los principales obstáculos para la formulación de políticas en salud adecuadas a las poblaciones urbanas marginadas es la carencia de información específica que describa las necesidades de salud de esos núcleos poblacionales. Aunque la pobreza urbana ha sido un tema de estudio que ha adquirido relevancia mundial - lo que se manifiesta en su inclusión en los Objetivos de Desarrollo del Milenio de la ONU como "mejoras significativas en la vida de al menos 100 millones de habitantes marginados de las ciudades"-, la definición de población marginada no ha sido establecida claramente.

En México, a pesar de los esfuerzos por sistematizar la definición de marginación mediante indicadores como los ingresos, la salud, la educación, la tenen- cia de la vivienda, y algunos indicadores de bienes o servicios en el hogar $(9,10)$, las encuestas nacionales de salud no ofrecen una desagregación suficiente que permita representar a la población de las áreas urbanas marginadas, lo que provoca que los problemas de salud de los grupos que residen en ellas no se puedan distinguir de los del resto del centro urbano.

Por ello, se requiere de una revisión específica de las necesidades de salud de esa población mediante el análisis de las condiciones de salud y de la situación particular de riesgo como indicadores de dichas necesidades (11) Esto permitirá tener elementos suficientes para la planeación estratégica de programas específicos dirigidos a su atención. Bajo esta perspectiva, el objetivo del presente trabajo fue evaluar las necesidades de salud de la población residente en áreas urbanas marginadas de México.

\section{MATERIALES Y MÉTODOS}

Entre septiembre de 2002 y febrero de 2003 se realizó una encuesta poblacional en colonias marginadas de ciudades mayores de 15000 habitantes de México.

Todos los integrantes de las familias que resultaron seleccionadas se dividieron en seis grupos de análisis según la edad y el sexo: niños (menores de 10 años), adolescentes hombres y mujeres (de 10 a 19 años), adultos hombres y mujeres (de 20 a 59 años) y adultos mayores (de 60 años o más). Para fines de este trabajo, la familia se definió como el conjunto de personas que hacen vida en común dentro de una vivienda, independientemente del parentesco, que comparten gastos de manutención y preparan los alimentos conjuntamente en la misma cocina.

La encuesta se diseñó para extraer inferencias a nivel nacional y para cinco regiones geográficas de México: Norte, Centro, Sur, Sureste y Ciudad de México (por ser la ciudad más poblada del país con casi 9 millones de habitantes, esta última se consideró como una región). Se realizó un muestreo polietápico en el que la unidad última de análisis fueron las personas de las familias seleccionadas por grupos de análisis. En la etapa I se llegó a la clasificación regional ya mencionada. Para la etapa II se formaron tres estratos de ciudades según su número de habitantes: estrato 1, de 15001 a 100 000; estrato 2, de 100001 a 500 000; estrato 3, más de 500 000. De cada región se seleccionaron mediante aleatorización simple tres ciudades, una de cada estrato, a partir de una lista de localidades mayores de 15000 habitantes de todo el país proporcionada específicamente para este estudio por el Instituto Nacional de Geografía e Informática. En la ciudad de México, los estratos estuvieron constituidos por las delegaciones políticas de acuerdo con el número de habitantes. Las ciudades seleccionadas para la región Norte por estado federativo fueron Guasave (Sinaloa), Saltillo (Coahuila) y Tijuana (Baja California). En la región Centro se seleccionaron Apaseo el Grande (Guanajuato), Orizaba (Veracruz) y Zapopan (Jalisco). La región Sur estuvo representada por Salina Cruz (Oaxaca), San Cristóbal de las Casas (Chiapas) y Acapulco (Guerrero). En la región del Sureste se seleccionaron Comalcalco (Tabasco), Campeche (Campeche) y Mérida (Yucatán). En el Distrito Federal, las delegaciones participantes fueron Tláhuac, Atzcapotzalco y Cuauhtémoc.

En la etapa III se eligieron de manera aleatoria las colonias o barrios marginados al interior de cada ciudad o delegación en los que faltara al menos uno de los siguientes servicios públicos: pavimento, energía eléctrica, drenaje y agua potable, según la información disponible en las coordinaciones estatales de Salud Pública del Instituto Mexicano del Seguro Social (IMSS). Se visitaron las colonias seleccionadas para confirmar su elegibilidad; en dos ciudades (Guasave y Distrito Federal) se sustituyeron las colonias por no cumplir los criterios de marginación. Para el levantamiento de la información se visitaron todas las viviendas a partir de un punto de la colonia tomado arbitrariamente hasta completar la cuota establecida para cada ciudad.

El tamaño de la muestra se determinó mediante el cálculo de la proporción en una población con precisión absoluta, de acuerdo con los siguientes supuestos (12): nivel de confianza de $95 \%$, proporción de la variable de interés de $50 \%$, precisión de $7 \%$, efecto de diseño de 2 y excedente por la tasa prevista de no respuesta de $20 \%$. El tamaño de la muestra final en cada ciudad seleccionada fue de 462 familias.

\section{Variables de estudio}

Se definieron variables indicadoras de las necesidades de salud por cada grupo 
de análisis y se agruparon en los siguientes seis dominios: salud positiva (percepción del estado de salud y vacunas recibidas), estado de nutrición, salud reproductiva, daños a la salud (accidentes $\mathrm{y}$ actos de violencia sufridos, enfermedades, discapacidad y adicciones), salud mental (depresión) y utilización de los servicios de salud. En este trabajo se presentan los resultados más relevantes de los cinco primeros dominios.

\section{Descripción del estudio}

Capacitación. Durante una semana se capacitó a enfermeras supervisoras - una por cada ciudad, residentes en la localidad y con experiencia en la aplicación de encuestas - en el levantamiento de la información y la medición del peso y la talla.

Instrumentos. Se elaboraron seis cuestionarios, uno por cada grupo de análisis. Los cuestionarios individuales recababan información acerca de los riesgos y los daños a la salud y sobre la utilización de servicios preventivos, ambulatorios y hospitalarios. Asimismo, en los mayores de 11 años se obtuvo información acerca de la percepción de su estado de salud y se les aplicó el Cuestionario Estructural Tetradimensional para la Depresión (CET-DE) (13) para detectar la presencia de síntomas de depresión a partir de la autoevaluación de cuatro dimensiones (humor depresivo, anergia, trastornos de comunicación y ritmopatía) con cuatro preguntas cada una. Se consideró a un individuo con depresión o características de depresión cuando el puntaje total en el cuestionario era de 8 puntos (de un total de 16) o más de 2 puntos en alguna de las dimensiones; la intensidad se valoró indirectamente por el número de dimensiones afectadas.

Material de trabajo. Para medir la talla de los niños menores de 24 meses de edad se utilizaron infantómetros de aluminio Seca 208 (Hamburgo, Alemania) y para el resto de los participantes, estadímetros Seca 214, ambos con una precisión de $1 \mathrm{~mm}$. Para la medición del peso se utilizaron básculas electrónicas Tanita 1582 (Tokio, Japón) con una precisión de $20 \mathrm{~g}$ para los menores de $14 \mathrm{~kg}$ y de $100 \mathrm{~g}$ para los mayores.

Levantamiento de la información. En una primera visita a las familias seleccionadas se realizó un censo de los inte- grantes de la familia y, previo consentimiento informado verbal, se aplicó la entrevista a quienes se encontraban en la vivienda y aceptaron dar respuesta en ese momento (los datos de los menores de 10 años se obtuvieron por entrevista indirecta con la madre o responsable del cuidado del niño). Cuando por ausencia de algún integrante de la familia no se obtuvo la totalidad de la información o de las mediciones, se concertó una cita para el día más conveniente para esa persona -incluso fines de semana- y se regresó a la vivienda las veces necesarias para concluir la encuesta. A cada integrante de la familia se le midió el peso y la talla. Los hogares que no aceptaron participar o las viviendas que se encontraron cerradas después de tres visitas se sustituyeron por los hogares consecutivos de la misma colonia (barrio) hasta completar la cuota establecida.

\section{Análisis de los datos}

El análisis se realizó mediante la determinación de las frecuencias absolutas y relativas para cada grupo de análisis. Para la evaluación del estado nutricional de los niños menores de 5 años se utilizaron los estándares de crecimiento propuestos por la Organización Mundial de la Salud (OMS) en 2006 (14). Para la clasificación del estado nutricional de los escolares se utilizaron dos criterios: con el propuesto por el Centro para el Control y la Prevención de Enfermedades (CDC) de los Estados Unidos de América (15) en 2000 se estimó la prevalencia de desnutrición a partir del índice de masa corporal (IMC), mientras que con el propuesto por el Grupo Internacional de Trabajo sobre la Obesidad (IOTF) (16) se calcularon las prevalencias de sobrepeso y de obesidad. En los adolescentes se utilizaron los criterios de la IOTF, mientras que en los adultos y adultos mayores, el estado de nutrición se clasificó de acuerdo con los criterios propuestos por la OMS en 1997 (17): desnutrición IMC $<18,5$, normal $25<$ IMC $\geq 18,5$, sobrepeso $30<$ IMC $\geq 25$ y obesidad IMC $\geq 30$.

Este proyecto fue aprobado por la Comisión Nacional de Investigación Científica de México y la Subcomisión de Ética del IMSS, con el registro No. 2002-785-005.

\section{RESULTADOS}

Se encuestaron 6658 familias y 24707 personas, distribuidas según los grupos de análisis de la siguiente forma: 6991 $(28,3 \%)$ niños, $5156(20,9 \%)$ adolescentes (2 569 mujeres y 2587 hombres), 6308 $(25,5 \%)$ mujeres adultas, $5160(20,9 \%)$ hombres adultos y $1092(4,4 \%)$ adultos mayores. Según el tamaño de la localidad, a las ciudades menores de 100000 habitantes correspondió $32,6 \%$ de la muestra, las de 100001 a 500000 habitantes aportaron el $32,9 \%$ y a las mayores de 500000 habitantes correspondió 34,5\%. La tasa general de no respuesta fue de $17,6 \%$, la mayoría correspondiente al grupo de hombres adultos, que estaban ausentes del domicilio durante las visitas realizadas. De la muestra total, $48,8 \%$ era del sexo masculino y $51,2 \%$ del femenino.

En el cuadro 1 se muestran algunas características sociodemográficas de la población de 12 años o más. De la población estudiada, 64,1\% tenía escolaridad de primaria o inferior; esta situación fue más grave en los adultos mayores, donde esa proporción fue de 93,9\% y 60,6\% nunca asistió a la escuela. En general, 24,5\% de las mujeres adultas y $27,1 \%$ de los hombres adultos dijeron vivir en unión libre; $29,4 \%$ de los adultos mayores eran viudos o viudas. En cuanto a la ocupación, $68,2 \%$ de los adolescentes estudiaba y en los grupos de mujeres adultas y adultos mayores predominaron las personas dedicadas a las labores del hogar; los hombres adultos se distribuyeron casi en la misma proporción en obreros $(27,7 \%)$, empleados $(22,3 \%)$ y personal de servicios (20,8\%). De los entrevistados, 53,2\% refirió no contar con seguro de salud público o privado.

La mayor parte $(63,5 \%)$ de las familias eran nucleares, mientras $29,3 \%$ eran consideradas extensas y 7,2\% correspondió a personas que vivían solas. Las características de sus viviendas reflejaron las condiciones de marginación de las áreas estudiadas: $22,5 \%$ tenía piso de tierra, $35,0 \%$ compartía el espacio para la cocina y el dormitorio y en $27,7 \%$ había hacinamiento. Solo 50,4\% recibía agua potable dentro del domicilio y $38,6 \%$ tenía drenaje (datos no presentados en los cuadros).

\section{Salud positiva}

La percepción del estado de salud varió de acuerdo con la edad; sin embargo, ya en los adolescentes se observaron algunos problemas, ya que $66,8 \%$ de ellos consideraba su salud como buena, casi la misma proporción que en el 
CUADRO 1. Características (\%) de la población urbana marginada de México a partir de 12 años de edad, septiembre de 2002 a febrero de 2003

\begin{tabular}{|c|c|c|c|c|c|}
\hline Característica & $\begin{array}{c}\text { Adolescentes } \\
\text { (de } 12 \text { a } 19 \text { años) } \\
(n=3914)\end{array}$ & $\begin{array}{c}\text { Mujeres } \\
\text { (de } 20 \text { a } 59 \text { años) } \\
(n=6308)\end{array}$ & $\begin{array}{c}\text { Hombres } \\
\text { (de } 20 \text { a } 59 \text { años) } \\
(n=5160)\end{array}$ & $\begin{array}{c}\text { Adultos mayores } \\
\text { (60 años o más) } \\
(n=1092)\end{array}$ & $\begin{array}{c}\text { Total } \\
(n=16474)\end{array}$ \\
\hline \multicolumn{6}{|l|}{ Escolaridad } \\
\hline Ninguna & 4,3 & 19,7 & 15,0 & 60,6 & 17,2 \\
\hline Primaria incompleta & 11,3 & 15,3 & 12,6 & 17,0 & 13,6 \\
\hline Primaria completa & 46,4 & 30,6 & 30,3 & 16,3 & 33,3 \\
\hline Secundaria & 30,1 & 22,9 & 27,3 & 3,1 & 24,8 \\
\hline Bachillerato & 7,9 & 8,8 & 10,4 & 2,0 & 8,6 \\
\hline Educación superior & 0,0 & 2,7 & 4,4 & 1,0 & 2,5 \\
\hline \multicolumn{6}{|l|}{ Estado civila } \\
\hline Casado & 7,4 & 50,3 & 54,4 & 44,4 & 44,9 \\
\hline Soltero & 82 & 15,3 & 15,3 & 5,1 & 24,7 \\
\hline Unión libre & 10,2 & 24,5 & 27,1 & 11,9 & 22,4 \\
\hline Viudo & 0,0 & 3,3 & 0,8 & 29,4 & 3,8 \\
\hline Divorciado/separado & 0,4 & 6,6 & 2,4 & 9,2 & 4,2 \\
\hline \multicolumn{6}{|l|}{ Ocupación } \\
\hline Trabajo de casa & 9,2 & 68,9 & 0,8 & 40,0 & 30,5 \\
\hline Campesino & 0,7 & 0,4 & 3,1 & 5,3 & 1,6 \\
\hline Servicios & 4,1 & 5,4 & 20,8 & 8,7 & 10,4 \\
\hline Empleado & 4,5 & 3,3 & 22,3 & 4,0 & 9,9 \\
\hline Obrero & 7,5 & 11,2 & 27,7 & 7,3 & 15,5 \\
\hline Comerciante & 0,9 & 4,1 & 6,8 & 7,5 & 4,4 \\
\hline Estudiante & 68,2 & 3,6 & 10,7 & 0,3 & 21,5 \\
\hline Jubilado/pensionado & 0,0 & 0,1 & 0,7 & 12,9 & 3,1 \\
\hline Desempleado & 4,9 & 1,0 & 3,5 & 6,8 & 1,1 \\
\hline Otra & 0,0 & 3,0 & 3,6 & 7,2 & 2,0 \\
\hline \multicolumn{6}{|l|}{ Seguro de salud } \\
\hline IMSS $^{b}$ & 27,5 & 34,6 & 35,4 & 42,9 & 35,1 \\
\hline ISSSTEC & 5,2 & 4,7 & 5,0 & 7,8 & 5,3 \\
\hline Otrod & 6,4 & 6,8 & 6,9 & 6,0 & 6,4 \\
\hline Ninguno & 60,9 & 53,9 & 52,7 & 43,3 & 53,2 \\
\hline
\end{tabular}

grupo de hombres adultos $(60,3 \%)$. Esta similitud se extendió a las categorías de regular $(30,2$ y $34,7 \%$, respectivamente) y mala (3,0 y 5,0\%, respectivamente). La percepción en las mujeres adultas fue menos favorable, ya que casi la mitad consideró su salud como regular $(43,7 \%)$ o mala $(8,4 \%)$, mientras que $74,8 \%$ de los adultos mayores se agrupó en estas categorías (50,8\% regular y $24,0 \%$ mala).

Con respecto a la cobertura de la vacunación, los esquemas correspondientes a los niños menores de 10 años (antituberculósica BCG; antivarólica de Sabin; antidifteria-pertusis-tétanos [DPT], DPTantiinfluenza o pentavalente, y triple viral) tuvieron una cobertura de 99,0\%. La cobertura para toxoide tetánico-diftérico fue de $66,0 \%$ en los adolescentes, $72,2 \%$ en las mujeres adultas, $64,0 \%$ en los hombres adultos y $46,1 \%$ en los adultos mayores; en estos últimos, la vacunación antiinfluenza y antineumocócica mostró coberturas de $13,3 \%$ y 13,8, respectivamente (datos no presentados en los cuadros).

\section{Nutrición}

Se obtuvo información antropométrica en $97,9 \%$ de la muestra. En los niños, la frecuencia de desmedro (talla baja para la edad) fue de 19,8\%, ligeramente mayor en los niños que en las niñas: $20,9 \%$ frente a $18,6 \%$, respectivamente (datos no mostrados en los cuadros). La prevalencia de desnutrición en los niños y niñas fue de $8,0 \%$, mientras que la prevalencia conjunta de sobrepeso y obesidad fue de 18,9\%. En los adolescentes, los adultos y los adultos mayores, la presencia de desnutrición fue muy baja, aunque la prevalencia conjunta de sobrepeso y obesidad en estos grupos de edad fue muy elevada $(24,7 \%, 63,5 \%$ y $66,6 \%$, respectivamente) y aumentó con la edad (cuadro 2).

\section{Salud sexual y reproductiva}

La información sobre este rubro sólo se investigó en los adolescentes (mujeres y hombres) mayores de 12 años y las mujeres adultas, por considerarlos los grupos más vulnerables en términos de necesidades de salud en este aspecto. Los datos más relevantes se presentan en el cuadro 3. De las 1984 adolescentes mujeres mayores de 12 años, $375(18,9 \%)$ tenían una vida sexualmente activa y de ellas, 20,5\% se encontraba embarazada al momento de realizar la encuesta. En el caso de los 1930 adolescentes hombres mayores de 12 años, 239 (12,4\%) declararon haber iniciado su vida sexual. Se encontró un bajo porcentaje de uso de anticonceptivos en la primera relación sexual de los adolescentes $(45,2 \%$ de los varones y solo $20,3 \%$ de las mujeres) y la proporción de infecciones de transmisión sexual informada fue de $7,5 \%$ en hombres y $8,8 \%$ en mujeres de este grupo de edad. De los adolescentes que al momento de la encuesta mencionaron utilizar anticonceptivos (ellos o sus parejas), el dispositivo intrauterino fue el más frecuentemente utilizado por parte 
CUADRO 2. Estado de nutrición (\%) de la población urbana marginada de México, septiembre de 2002 a febrero de 2003

\begin{tabular}{lccccc}
\hline Grupo de edad y sexo & No. $^{\mathrm{a}}$ & Desnutrición $^{\mathrm{b}}$ & Normal & Sobrepeso $^{\mathrm{c}}$ & Obesidad $^{\mathrm{d}}$ \\
\hline De 0 a 9 años & & & & & \\
$\quad$ Masculino & 3485 & 8,5 & 72,0 & 11,4 & 8,0 \\
$\quad$ Femenino & 3307 & 7,4 & 74,1 & 11,1 & 7,4 \\
$\quad$ Total & 6792 & 8,0 & 73,1 & 11,2 & 7,7 \\
De 10 a 19 años & & & & & \\
$\quad$ Masculino & 2556 & 1,2 & 74,1 & 10,0 & 14,6 \\
$\quad$ Femenino & 2536 & 0,6 & 74,8 & 11,0 & 13,6 \\
$\quad$ Total & 5092 & 0,9 & 74,4 & 10,6 & 14,1 \\
De 20 a 59 años & & & & & \\
Masculino & 5051 & 1,4 & 38,6 & 41,6 & 18,4 \\
$\quad$ Femenino & 6223 & 1,5 & 32,2 & 35,9 & 30,5 \\
$\quad$ Total & 11274 & 1,5 & 35,0 & 38,4 & 25,1 \\
$\geq 60$ años & & & & & \\
$\quad$ Masculino & 466 & 1,9 & 35,8 & 41,2 & 21,0 \\
$\quad$ Femenino & 557 & 2,1 & 27,5 & 33,9 & 36,4 \\
Total & 1023 & 2,0 & 31,3 & 37,2 & 29,4 \\
\hline
\end{tabular}

${ }^{a}$ Número de personas de quienes se obtuvo información antropométrica.

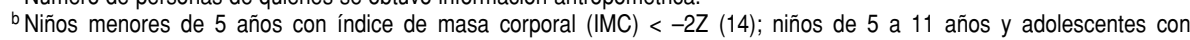
IMC < percentil 5 (15); adultos con índice IMC $<18,5$.

${ }^{c}$ Niños menores de 5 años con IMC > $2 Z$ (14); para niños de 5 a 11 años y adolescentes se utilizó el IMC de referencia por sexo y edad (16); adultos con $30 \leq \mathrm{IMC}>25$.

d Niños menores de 5 años con IMC > $3 Z$ (14); para niños de 5 a 11 años y adolescentes se utilizó el IMC de referencia por sexo y edad (16); adultos con IMC > 30 .

CUADRO 3. Características y prácticas de los adolescentes y las mujeres adultas con vida sexual activa en la población urbana marginada de México, septiembre de 2002 a febrero de 2003

\begin{tabular}{|c|c|c|c|c|}
\hline Característica/práctica & $\begin{array}{c}\text { Mujeres } \\
\text { adolescentes } \\
\text { (de } 12 \text { a } 19 \text { años) } \\
(n=375)\end{array}$ & $\begin{array}{c}\text { Hombres } \\
\text { adolescentes } \\
\text { (de } 12 \text { a } 19 \text { años }) \\
(n=239)\end{array}$ & $\begin{array}{c}\text { Mujeres } \\
\text { adultas } \\
\text { (de } 20 \text { a } 59 \text { años }) \\
(n=4604)\end{array}$ & $\begin{array}{c}\text { Total } \\
(n=5218)\end{array}$ \\
\hline $\begin{array}{l}\text { Mediana de la edad del } \\
\text { inicio sexual (mínimo- } \\
\text { máximo), años }\end{array}$ & $16(11-19)$ & $16(11-19)$ & $18(10-40)$ & $18(10-40)$ \\
\hline $\begin{array}{l}\text { Uso de anticonceptivo en } \\
\text { la primera relación (\%) }\end{array}$ & 20,3 & 45,2 & $\mathrm{NE}^{\mathrm{a}}$ & 30,3 \\
\hline $\begin{array}{l}\text { Antecedentes de infección } \\
\text { de transmisión sexual (\%) }\end{array}$ & $\begin{array}{r}8,8 \\
20\end{array}$ & $\begin{array}{r}7,5 \\
N^{b}\end{array}$ & $\begin{array}{r}18,1 \\
7 ?\end{array}$ & $\begin{array}{r}17,0 \\
8 ?\end{array}$ \\
\hline $\begin{array}{l}\text { Embarazada (\%) } \\
\text { Uso actual de }\end{array}$ & 20,5 & $N A^{b}$ & 7,2 & 8,2 \\
\hline $\begin{array}{c}\text { anticonceptivos }^{\mathrm{c}}(\%) \\
\text { Método anticonceptivo } \\
\text { ( }(\%)\end{array}$ & $\begin{array}{c}57,7 \\
(n=146)\end{array}$ & $\begin{array}{c}41,9 \\
(n=60)\end{array}$ & $\begin{array}{c}74,1 \\
(n=3410)\end{array}$ & $\begin{array}{c}69,3 \\
(n=3616)\end{array}$ \\
\hline Condón & 27,8 & 60,7 & 5,9 & 22,0 \\
\hline Dispositivo intrauterino & 39,6 & 13,1 & 21,5 & 22,0 \\
\hline Hormonales & 24,1 & 18,0 & 14,1 & 14,6 \\
\hline Salpingoclasia/vasectomía & 2,4 & 0,0 & 48,7 & 46,7 \\
\hline Otro/No especificado & 6,3 & 8,2 & 9,3 & 9,1 \\
\hline
\end{tabular}

${ }^{a} \mathrm{NE}$ : no evaluado.

bNA: no aplicable.

c Se excluyeron las embarazadas y quienes mencionaron no tener pareja en el momento de la entrevista.

¿Se incluyó a la pareja.

de las mujeres adolescentes $(39,6 \%)$, aunque $24,1 \%$ de ellas dijo utilizar métodos hormonales y $27,8 \%$ prefería que su pareja usara condones. En cuanto a los hombres adolescentes, 60,7\% dijo usar preservativos. Se debe resaltar que de las mujeres adultas que usaron algún método anticonceptivo, 48,7\% empleó la salpingoclasia (cuadro 3).

\section{Daños a la salud}

Entre las variables estudiadas solamente en el grupo de niños, se encontró una prevalencia lápsica (enfermedad durante las dos semanas previas a la entrevista) de infecciones respiratorias agudas de 39,5\% y de enfermedad diarreica aguda de $11,9 \%$. La frecuencia de niños con alguna deficiencia del lenguaje fue de $7,7 \%$, mientras que las dificultades para el aprendizaje y los trastornos de la visión y la audición fueron menores $(4,1 \%, 2,6 \% \mathrm{y}$ $1,6 \%$, respectivamente) (datos no mostrados en los cuadros).

De los adultos, 3,4\% de las mujeres y $6,8 \%$ de los hombres refirieron haber sufrido accidentes en el último año (cuadro 4). En todos los grupos, las caídas ocuparon el primer lugar y representaron más de la mitad de todos los accidentes, excepto en los hombres adultos, en quienes las caídas fueron la causa de $38,7 \%$ y los de transporte terrestre 27,2\%. En cuanto a la violencia en el mismo período, $3,2 \%$ de los hombres adultos informaron haber sido víctimas de actos violentos, principalmente por asaltos $(63,5 \%)$, mientras que la frecuencia de violencia en las mujeres adultas fue de 1,8\% y las agresiones sin motivo aparente ocurrió en el $30,7 \%$ de esos casos. En los adolescentes que mencionaron haber sufrido violencia en el último año, 29,5\% correspondió a asaltos, agresiones sin motivo aparente y discusiones.

La proporción total de personas con diabetes mellitus tipo 2 fue de 5,9\% y $11,5 \%$ de los entrevistados afirmó sufrir de hipertensión arterial, con la mayor frecuencia en adultos mayores $(28,1 \%)$ y mujeres adultas $(13,2 \%)$. Por último, 2,3\% de la muestra y 5,3\% de los adultos mayores tenían alguna discapacidad. En cuanto a la salud mental, 20,2\% de toda la población manifestó tener depresión en al menos una de las cuatro dimensiones analizadas (humor depresivo, anergia, trastornos de la comunicación y ritmopatía) y 1,8\% consideraba tener afectaciones en las cuatro dimensiones. Los grupos más afectados fueron las mujeres adultas y los adultos mayores (cuadro 4).

De los mayores de 10 años, 53,1\% mencionó haber fumado en alguna ocasión y 72,1\% de ellos lo hizo por primera vez antes de cumplir 20 años; 21,2\% de los participantes fumaba al momento de la encuesta (cuadro 5), de ellos 29,7\% fumaba más de cinco cigarrillos diarios (datos no mostrados). El consumo de bebidas alcohólicas en el momento de la encuesta lo refirieron $21,6 \%$ de los adolescentes, 25,9\% de las mujeres adultas, $64,8 \%$ de los hombres adultos y $25,6 \%$ de los adultos mayores y el consumo diario fue de $5,5 \%, 0,4 \%, 2,5 \%$ y 1,8\%, respectivamente (cuadro 5 ).

Por último, 9,5\% de la población mayor de 10 años de edad había consu- 
CUADRO 4. Problemas de salud (\%) en la población urbana marginada de México, septiembre de 2002 a febrero de 2003

\begin{tabular}{|c|c|c|c|c|c|c|}
\hline Problema de salud & $\begin{array}{c}\text { Niños } \\
\text { (de } 0 \text { a } 9 \text { años) } \\
(n=6991)\end{array}$ & $\begin{array}{c}\text { Adolescentes } \\
\text { (de } 10 \text { a } 19 \text { años) } \\
(n=5156)\end{array}$ & $\begin{array}{c}\text { Mujeres } \\
\text { (de } 20 \text { a } 59 \text { años) } \\
(n=6308)\end{array}$ & $\begin{array}{c}\text { Hombres } \\
\text { (de } 20 \text { a } 59 \text { años) } \\
(n=5160)\end{array}$ & $\begin{array}{l}\text { Adultos mayores } \\
\text { (60 años o más) } \\
(n=1092)\end{array}$ & $\begin{array}{c}\text { Total } \\
(n=24707)\end{array}$ \\
\hline Accidentes $^{a}$ & 5,3 & 5,0 & 3,4 & 6,8 & 6,7 & 5,1 \\
\hline Víctima de violencia ${ }^{a}$ & $\mathrm{NE}^{\mathrm{b}}$ & 1,7 & 1,8 & 3,2 & 0,9 & 2,1 \\
\hline Diabetes tipo 2 & $\mathrm{NE}$ & NE & 5,2 & 3,5 & 18,0 & 5,9 \\
\hline Hipertensión & NE & NE & 13,2 & 5,4 & 28,1 & 11,5 \\
\hline Discapacidad & 1,2 & 2,6 & 2,3 & 2,8 & 5,3 & 2,3 \\
\hline Depresión en una o más dimensiones ${ }^{C}$ & NE & $16,8^{d}$ & 25,5 & 13,3 & 37,8 & 20,2 \\
\hline Depresión en las cuatro dimensiones ${ }^{c}$ & NE & $0,9^{d}$ & 2,7 & 0,8 & 4,9 & 1,8 \\
\hline
\end{tabular}

\footnotetext{
a En el último año.

b NE: no evaluado.

${ }^{c}$ Las dimensiones fueron: humor depresivo, anergia, trastornos de comunicación y ritmopatía.

${ }^{\mathrm{d}}$ Adolescentes a partir de 11 años $(n=4$ 038).
}

CUADRO 5. Adicciones (\%) en la población urbana marginada de México, septiembre de 2002 a febrero de 2003

\begin{tabular}{|c|c|c|c|c|c|}
\hline Adicción & $\begin{array}{c}\text { Adolescentes } \\
\text { (de } 10 \text { a } 19 \text { años) } \\
(n=5156)\end{array}$ & $\begin{array}{c}\text { Mujeres } \\
\text { (de } 20 \text { a } 59 \text { años) } \\
(n=6308)\end{array}$ & $\begin{array}{c}\text { Hombres } \\
\text { (de } 20 \text { a } 59 \text { años) } \\
(n=5160)\end{array}$ & $\begin{array}{c}\text { Adultos mayores } \\
\text { (60 años o más) } \\
(n=1092)\end{array}$ & $\begin{array}{c}\text { Total } \\
(n=17716)\end{array}$ \\
\hline Ha fumado alguna vez & 12,3 & 26,7 & 73,5 & 38,8 & 53,1 \\
\hline \multicolumn{6}{|l|}{ Edad a la que fumó por primera vez } \\
\hline$<16$ años & 7,3 & 5,2 & 25,7 & 10,4 & 17,3 \\
\hline De 16 a 19 años & 5,0 & 9,3 & 32,7 & 9,0 & 21,2 \\
\hline$\geq 20$ años & 0,0 & 12,3 & 15,1 & 19,4 & 14,7 \\
\hline Tabaquismo actual & 12,3 & 10,8 & 44,0 & 15,8 & 21,2 \\
\hline \multicolumn{6}{|l|}{ Número de cigarrillos que fuma actualmente } \\
\hline De 1 a 5 & 9,5 & 7,9 & 30,1 & 8,8 & 14,9 \\
\hline De 6 a 10 & 0,6 & 1,1 & 6,2 & 3,6 & 2,6 \\
\hline De 11 a 20 & 0,3 & 0,6 & 4,3 & 2,5 & 1,7 \\
\hline$>20$ & 0,1 & 1,0 & 3,1 & 1,0 & 1,3 \\
\hline No especificado & 1,8 & 0,2 & 0,3 & 0,0 & 0,7 \\
\hline Consumo actual de bebidas alcohólicas & 21,6 & 25,9 & 64,8 & 25,6 & 36,0 \\
\hline \multicolumn{6}{|c|}{ Frecuencia de consumo de bebidas alcohólicas } \\
\hline Diario & 5,5 & 0,4 & 2,5 & 1,8 & 2,7 \\
\hline Ocasional & 15,3 & 24,8 & 62,3 & 23,0 & 32,8 \\
\hline No especificado & 0,8 & 0,6 & 0,0 & 0,8 & 0,5 \\
\hline Ha consumido drogas alguna vez & 2,7 & 10,1 & 15,0 & 11,2 & 9,5 \\
\hline \multicolumn{6}{|l|}{ Droga consumida } \\
\hline Tranquilizantes & 1,3 & 10,3 & 7,1 & 14,9 & 6,9 \\
\hline Anfetaminas & 0,5 & 3,0 & 1,2 & 1,1 & 1,6 \\
\hline Marihuana & 1,0 & 0,3 & 8,1 & 0,9 & 2,8 \\
\hline Cocaína & 0,4 & 0,3 & 3,7 & 0,3 & 1,3 \\
\hline Inhalantes & 0,5 & 0,2 & 1,9 & 0,0 & 0,8 \\
\hline Otras & 0,2 & 0,2 & 1,3 & 0,5 & 0,5 \\
\hline
\end{tabular}

mido drogas $(2,7 \%$ de los adolescentes, $10,1 \%$ de las mujeres adultas, $15,0 \%$ de los hombres adultos y $11,2 \%$ de los adultos mayores). Los tranquilizantes fueron los más frecuentemente mencionados; en el grupo de hombres adultos hubo una mayor diversidad de drogas, entre ellas la marihuana, las drogas inhaladas, la cocaína y las anfetaminas (cuadro 5).

\section{DISCUSIÓN}

La población estudiada mostró una composición más joven que la media na- cional, en la que la tercera parte corresponde a menores de 15 años, casi $10 \%$ menos que en este estudio. Sin embargo, la proporción de personas de 70 años o más fue similar (3,6 frente a 2,0\%, respectivamente) (18). Esta composición de la población, así como la de las familias - en su mayoría nucleares-, es la esperada si se toma en cuenta que los barrios marginados de las ciudades están integrados por familias nuevas, muchas de ellas procedentes de otras localidades y que se establecen de manera aislada (3). Esto representa una primera condición de vulnerabilidad para la población joven, que carece de redes de apoyo familiar y tiene la necesidad de sobrevivir en un ambiente social adverso. A pesar de ello y de la baja escolaridad de los grupos en edad productiva, llama la atención la baja proporción de desempleo encontrada ( $4,8 \%$ en hombres adultos), menor que lo informado en 2004 para las áreas urbanas (19), aunque el criterio para calificar el desempleo en esta encuesta fue el autoinforme. Una posible explicación es que la mayoría de las personas con empleo eran obreros o trabajadores de servi- 
cios, ocupaciones generalmente mal remuneradas y en muchas ocasiones eventuales; este dato también es congruente con la elevada proporción de familias sin seguro de salud.

Uno de los elementos más importantes para estimar la vulnerabilidad de las familias son las condiciones de salud de los niños (6). Como se ha documentado en otros países de América Latina (20), el estado nutricional de los niños constituye un indicador importante que refleja la inadecuada ingesta alimentaria cuando no se satisfacen las necesidades básicas; los resultados del presente estudio son una muestra de ello. El desmedro en uno de cada cinco niños casi duplica el nivel nacional de $12,7 \%$ en los menores de 5 años y de $10 \%$ en los escolares, según la Encuesta Nacional de Salud y Nutrición de 2006 (21). Por otro lado, el sobrepeso y la obesidad en este grupo es un reflejo de la mala nutrición en general, aunque la proporción encontrada está por debajo de la media en la población nacional (21).

Los adolescentes, como grupo poblacional, han recibido poca atención en los programas de salud, posiblemente por constituir un sector en el que ya se han superado los riesgos de enfermedad propios de la niñez y aún no presentan las condiciones que propician los problemas crónicos propios de la adultez. Si bien en los adolescentes se ha observado una proporción preocupante de sobrepeso y obesidad, en los que viven en áreas marginadas se han documentado otros riesgos adicionales que ameritan especial atención $(22,23)$. El haber encontrado una frecuencia de consumo de alcohol y tabaco mayor de la informada por la Encuesta Nacional de Adicciones (24) y la aceptación de casi 3\% de los adolescentes entrevistados de haber consumido drogas, son indicadores de la necesidad de una mayor atención a este grupo de edad en general. Lo mismo puede decirse con respecto al riesgo reproductivo, ya que la baja proporción de uso de métodos de protección los expone a enfermedades de transmisión sexual y embarazos no deseados y de alto riesgo, como se ha encontrado en otros estudios realizados en poblaciones similares (25). En este sentido, también se deben tomar en cuenta las consecuencias sociales para la mujer -en términos de desigualdad de género- derivadas del embarazo pre$\mathrm{COZ}$, como el truncamiento de la educación y las dificultades para su desarrollo personal debido a su involucramiento en las tareas reproductivas.

Aunque la salud es un proceso dinámico que resulta de la compleja interrelación de los factores biológicos, socioeconómicos, culturales y psicológicos, el peso de estos factores no se distribuye de la misma manera en todos los individuos a lo largo de su vida. Así, en las áreas pobres de las ciudades, las mujeres constituyen una población vulnerable por las diferencias de género relacionadas con la reproducción y el cuidado de los hijos y la discriminación que enfrentan, lo que se manifiesta en detrimento de su salud (6). Un análisis derivado de los datos de la presente encuesta mostró que 22,3\% de las mujeres mayores de 15 años fungía como jefa de su hogar (26), lo que refleja una elevada proporción de mujeres responsables de su familia desde el punto de vista económico y probablemente muchas de ellas también del cuidado de sus hijos y de su hogar. Además, se ha confirmado que las mujeres de estratos socioeconómicos desprotegidos tienen más dificultades para acceder a los servicios de salud (27). Todos estos elementos seguramente se manifiestan en la percepción encontrada sobre su estado de salud, que menos de la mitad de las mujeres adultas consideró buena, a diferencia de los hombres adultos que consideraron tener buen estado de salud en una mayor proporción $(60,3 \%)$. Indicadores objetivos, como la prevalencia de enfermedades crónicas, confirman la desventajosa condición de las mujeres con respecto a los hombres, aunque pareciera que son menos susceptibles a los daños por accidentes, la violencia y las adicciones. Un resultado interesante de la presente investigación es la alta proporción de mujeres adultas que prefirieron los métodos definitivos de anticoncepción, lo que amerita futuros estudios a fin de conocer el significado de dicho hallazgo en términos de cambios culturales tradicionales en este contexto social de México (28).

Por otro lado, los hombres en edad laboral enfrentan otro tipo de riesgos. Este grupo constituye el principal sustento de la mayoría de los hogares, por lo que sus condiciones de salud son fundamentales para definir el bienestar de todo el núcleo familiar. Sus riesgos se manifiestan principalmente por la exposición a la violencia, que fue mayor que la encontrada en este grupo por la Encuesta Nacional de Salud 2000 (2,4\%). La frecuen- cia y el tipo de accidentes fueron similares en ambas encuestas, aunque el consumo de alcohol y tabaco en los hombres adultos que viven en estas áreas marginadas fue más del doble que la media nacional en este grupo poblacional (29).

Por su parte, la población urbana marginada de 60 años o más es un núcleo poblacional de especial interés para la transición en salud. Este es un grupo vulnerable compuesto por personas cuyas condiciones de vida durante la infancia, la adolescencia y la adultez probablemente estuvieron marcadas por la desventaja social; muestra de ello es que más de la mitad de los adultos mayores entrevistados carecía de escolaridad. Esta desventaja social también implica poca atención a su estado de salud actual, ya sea por desconocimiento o por la insuficiente comprensión de su importancia, lo que dificulta la atención de sus problemas de salud, incluso a pesar de tener acceso a esos servicios (30). En el presente estudio, el perfil de salud de la población adulta mayor mostró prevalencias de diabetes y obesidad similares a las encontradas en el mismo grupo de edad de la población mexicana $(21,29)$; la prevalencia de hipertensión arterial fue menor que la media nacional $(28,1 \%$ frente a $53,2 \%$, respectivamente) (29) y a la encontrada en áreas urbanas de otros países de América Latina, que va de $43 \%$ a $53 \%$ (31). Aunque estos hallazgos deben evaluarse con investigaciones más detalladas que confirmen la precisión de los autoinformes, los resultados presentados confirman que las necesidades de salud de los adultos mayores que residen en zonas urbanas marginadas de México hacen de este grupo una prioridad para los servicios de salud.

Un resultado que se debe resaltar es el relacionado con el área de salud mental, principalmente en las mujeres adultas y los adultos mayores, que manifestaron elevadas frecuencias de síntomas depresivos, aunque en menor grado que en otros países de la región como Chile (32). Los datos de depresión mayor, manifestados por síntomas en las cuatro dimensiones analizadas, coinciden con otros estudios realizados en México (33), aunque resultaron menos frecuentes que en Colombia (34). Sin embargo, estas comparaciones deben ser tomadas con cautela debido a la diversidad de instrumentos empleados para la evaluación de la salud mental en los estudios citados. Asimismo, y como en todas las encuestas 
por entrevista, la confiabilidad de las estimaciones basadas en autoinformes es limitada, en particular por errores de subregistro en preguntas consideradas sensibles para determinados grupos, como el uso de drogas en adolescentes.

Una limitación a tomar en cuenta para la interpretación de estos resultados es el uso de indicadores que pueden afectar a determinados resultados, como la medición del estado nutricional en los adultos mayores mediante el IMC. Aunque debe admitirse la complejidad para lograr una correcta estimación de la situación nutricional en este grupo de edad (35), el IMC es el indicador recomendado por la OMS para generar las estadísticas poblacionales, incluidos los adultos mayores (36), aún a pesar de no contar con valores de referencia específicos que tomen en cuenta sus características anatómicas y fisiológicas. Se requieren estudios adicionales en el área de la nutrición geriátrica, particularmente en poblaciones vulnerables, para profundizar en este tema.

En cuanto a la representatividad de la muestra, si bien el diseño muestral per- mite una aproximación a la situación de las condiciones de salud de la población a niveles nacional y regional por estrato de ciudades, aún al interior del mismo estrato pueden existir condiciones particulares en determinadas ciudades que difieran de las seleccionadas. Asimismo, los estratos empleados no se ponderaron según el número de localidades existentes en el país, lo que debe tenerse presente al interpretar estos resultados.

En conclusión, la vulnerabilidad sanitaria de las personas que viven en las áreas urbanas marginadas de México se manifiesta en la existencia de una población predominantemente joven con pocas redes de apoyo familiar y de servicios de salud, que sufre trastornos y enfermedades producto del rezago en el desarrollo social, como la desnutrición infantil, el alto riesgo reproductivo y la adicción en adolescentes y adultos. No obstante, la prevalencia de enfermedades crónicas fue similar a la encontrada en la población mexicana no marginada.

Este estudio representa uno de los primeros esfuerzos por identificar las nece- sidades de salud de la población urbana marginada y describe las condiciones de un conjunto poblacional con características singulares, diferentes a las de las poblaciones urbanas o rurales en general. Estos resultados dan cuenta de un espectro muy amplio de problemas de salud y ponen de manifiesto el enorme desafío al que se enfrenta el sector salud en su conjunto. Se recomienda a los programas de protección social en salud que entre sus prioridades incluyan problemas poco atendidos y de alta prevalencia como los aquí referidos, con la finalidad de focalizar sus contenidos de acuerdo con estas realidades. Asimismo, estos programas deben evaluar de manera específica los beneficios obtenidos por esta población como resultado de los apoyos económico, nutricional, educativo o de servicios de salud que reciben.

Agradecimientos. Este proyecto recibió financiamiento del IMSS a través del Fondo para el Fomento de la Investigación (FOFOI) de la Coordinación de Investigación en Salud (No. FP-0038).

\section{REFERENCIAS}

1. United Nations. World population prospects: the 2005 revision. New York: United Nations; 2006. (Working Paper No. ESA/P/WP/200.)

2. Vlahov D, Galea S, Gibble E, Freudenberg N. Perspectives on urban conditions and population health. Cad Saude Publica. 2005;21(3): 949-7.

3. Dufour DL, Piperata BA. Rural-to-urban migration in Latin America: an update and thoughts on the model. Am J Hum Biol. 2004; 16(4):395-4.

4. Chen JT, Rehkopf DH, Waterman PD, Subramanian SV, Coull BA, Cohen B, et al. Mapping and measuring social disparities in premature mortality: the impact of census tract poverty within and across Boston neighborhoods, 1999-2001. J Urban Health. 2006;83(6): 1063-84.

5. Cohen DA, Farley TA, Mason K. Why is poverty unhealthy? Social and physical mediators. Soc Sci Med. 2003;57(9):1631-41.

6. Montgomery MR, Hewett PC. Urban poverty and health in developing countries: household and neighborhood effects. New York: Population Council; 2004. (Working Paper No. 184.)

7. Schneiders J, Drukker M, van der Ende J, Verhulst FC, van Os J, Nicolson NA. Neighbourhood socioeconomic disadvantage and behavioural problems from late childhood into early adolescence. J Epidemiol Community Health. 2003;59:699-703.
8. Vlahov D, Galea S. Urbanization, urbanicity, and health. J Urban Health. 2002;79(4 Suppl1): S1-12.

9. Consejo Nacional de Población. Índices de marginación 2000 [sitio en Internet]. México, D.F.: Secretaría de Gobernación; 2001. Hallado en http:/ / www.conapo.gob.mx/00 cifras / 2000.htm. Acceso el 18 de marzo de 2009.

10. Brambila C. La pobreza urbana en México. Borrador para comentarios. México, D.F.: Instituto Tecnológico de Monterrey; 2006. (Documento de trabajo EGAP-2006-13). Hallado en http://alejandria.ccm.itesm.mx/egap/ documentos/EGAP-2006-13.pdf. Acceso el 10 de marzo de 2009.

11. Cavanagh S, Chadwick K. Health needs assessment: a practical guide. London: National Institute for health and Clinical Excellence; 2005. Hallado en http://www.nice.org.uk/ aboutnice/whoweare/aboutthehda/hdapu blications/health_needs_assessment_a_practi cal_guide.jsp. Acceso el 10 de marzo de 2009.

12. Lwanga SK, Lemeshow S. Sample size determination in health studies. Geneva: World Health Organization; 1991.

13. Alonso-Hernández F. Cuestionario estructural tetradimensional para la depresión. 3. ${ }^{\mathrm{a}}$ ed. Madrid: TEA Ediciones; 1995.

14. World Health Organization. WHO child growth standards. Geneva: World Health Organization; 2006. Hallado en http://www. who.int/growthref/who2007_bmi_for_age/ en/index.html. Acceso el 10 de marzo de 2009.

15. Ogden C, Kucszmarski R, Flegal K, Mei Z, Guo S, Wei R, et al. Centers for Disease Control and Prevention 2000 growth charts for the United States: improvements to the 1977 National Center for Health Statistics version. Pediatrics. 2002;109:45-60.

16. Cole TJ, Bellizzi MC, Flegal KM, Dietz WH. Establishing a standard definition for child overweight and obesity worldwide: international survey. Br Med J. 2000;320:1-6.

17. World Health Organization. Obesity: preventing and managing the global epidemic. Geneva: WHO; 2000. (WHO Technical Report Series No. 894.)

18. Instituto Nacional de Estadística, Geografía e Informática. Conteo de población y vivienda 2005. México, D.F.: INEGI; 2005. Hallado en http://www.inegi.org.mx/inegi/default.asp $\mathrm{x}$ ? $\mathrm{s}=\mathrm{est} \& \mathrm{c}=10382 \& \mathrm{e}=\& \mathrm{i}=$. Acceso el $10 \mathrm{de}$ marzo de 2009.

19. Instituto Nacional de Estadística, Geografía e Informática. Encuesta Nacional de Empleo Urbano 2005. México, D.F.: INEGI; 2005. Hallado en http://www.inegi.org.mx/inegi/ default.aspx?s=est\&c=10730\&e $=\& \mathrm{i}=$. Acceso el 10 de marzo de 2009.

20. Rojas DC, Calderón MP, Taipe MC, Bernui I, Ysla M, Riega V. Consumo de energía y nutrientes, características socioeconómicas, pobreza y área de residencia de niños peruanos 
de 12 a 35 meses de edad. Rev Peru Med Exp Salud Publica. 2004;21(2):98-106.

21. Olaíz-Fernández G, Rivera-Dommarco J, Shamah-Levy T, Villalpando-Hernández $\mathrm{S}$, Hernández-Ávila M, Sepúlveda-Amor J. Encuesta Nacional de Salud y Nutrición 2006. Cuernavaca, México: Instituto Nacional de Salud Pública; 2006.

22. Cubbin C, Santelli J, Brindis CD, Braveman P. Neighborhood context and sexual behaviors among adolescents: findings from the national longitudinal study of adolescent health. Perspect Sex Reprod Health. 2005; 37(3):125-34.

23. Lee RE, Cubbin C. Neighborhood context and youth cardiovascular health behaviors. Am J Public Health. 2002;92:428-36.

24. Consejo Nacional Contra las Adicciones. Encuesta Nacional de Adicciones (ENA) 2002. México, D.F.: CONADIC; 2002.

25. Gutiérrez JP, Bertozzi SM, Conde-González CJ, Sánchez-Alemán MA. Risk behaviors of 15-21 year olds in Mexico link to a high prevalence of sexually transmitted infections: results of a survey in disadvantaged urban areas. BMC Public Health. 2006;6:49. Hallado en http://www.biomedcentral.com/14712458/6/49. Acceso el 18 de marzo de 2009.
26. Torres Arreola L, Gómez Dantés H, Reyes Morales H, Tomé P, Vázquez Martínez JL. Necesidades de salud y jefatura del hogar en áreas urbano marginadas. Boletín Género y Salud en Cifras (Secretaría de Salud de México). 2002;2(2):13-20.

27. Lucumí Cuesta DI, Gómez Gutiérrez LF. Accesibilidad a los servicios de salud en la práctica de citología de cuello uterino en una zona urbana de Colombia. Rev Esp Salud Publica. 2004;78:367-77.

28. Ariza M, de Oliveira O. Familias en transición y marcos conceptuales en redefinición. Papeles Poblac. 2001;28:9-39.

29. Olaiz G, Barquera S, Shamah T, Aguilar C, Cravioto P, López P, et al. Encuesta Nacional de Salud 2000. Tomo 2. La salud de los adultos. Cuernavaca, México. Instituto Nacional de Salud Pública; 2003.

30. Fiscella K, Williams DR. Health disparities based on socioeconomic inequities: implications for urban health care. Acad Med. 2004; 79(12):1139-47.

31. Peláez M, Palloni A, Pinto G, Arias E. Encuesta multicéntrica: Salud, Bienestar y Envejecimiento (SABE). Washington, D.C.: Organización Panamericana de la Salud; 2001.
32. Pontificia Universidad Católica de Chile, Ministerio de Salud. Encuesta Nacional de Salud Chile 2003. Informe final. Santiago: MINSAL; 2004.

33. Medina-Mora ME, Borges $G$, Lara C, Benjet $C$, Blanco J, Fleiz C, et al. Prevalencia de trastornos mentales y uso de servicios: resultados de la Encuesta Nacional de Epidemiología Psiquiátrica en México. Salud Mental. 2003; 26(4)1-16.

34. Gómez-Restrepo C, Bohórquez A, Pinto Masis D, Gil Laverde JFA, Rondón Sepúlveda M, Díaz-Granados N. Prevalencia de depresión y factores asociados con ella en la población colombiana. Rev Panam Salud Publica. 2004; 16(6):378-86.

35. Lipschitz DA. Screening for nutritional status in the elderly. Prim Care. 1994;21(1):55-7.

36. World Health Organization. Global Database on body mass index. An interactive surveillance tool for monitoring nutrition transition. Geneva: WHO; 2006. Hallado en http:// www.who.int/bmi/index.jsp. Acceso el 10 de marzo de 2009.

Manuscrito recibido el 13 de diciembre de 2007. Aceptado para publicación, tras revisión, el 5 de septiembre de 2008.

ABSTRACT Objective. To understand the health needs of the population living in Mexico's marginalized urban areas.

\section{Health needs in marginalized urban areas in Mexico}

Key words Poverty areas, needs assessment, health status, Mexico.
Methods. A population-based survey of families residing in poor, urban neighborhoods, in five geographic areas in Mexico (northern, central, southern, south-east, and Mexico City), selected through multistage sampling. Interviews were conducted and anthropometric measurements were taken in the home, and included all members of the participating family. Analysis was carried out on positive health factors, nutrition, reproductive health, health problems, and mental health, and results were disaggregated by age and sex.

Results. In all, 24707 individuals participated. The interviewees were found to have minimal schooling (6 years or less). Only $46.8 \%$ had health care coverage, be it public or private. Among the children, $19.8 \%$ were malnourished; overweight was prevalent from adolescence onwards. Of adolescents 12-19 years of age, 15.7\% were sexually active, but only $57.7 \%$ of the males and $41.9 \%$ of the females in this age group were using some method of contraception. Of the adults, $5.9 \%$ suffered from diabetes and $11.5 \%$, from high blood pressure. In the sample of adolescents, adults, and elderly adults, tobacco use was $21.2 \%$; alcohol consumption, $36.0 \%$; illicit drug use, $9.5 \%$; and depression, $20.2 \%$.

Conclusions. The health needs of people living in Mexico's marginalized urban areas proliferate in the context of a young population with weak ties and little support from family and health services. They face crisis and disease-infant malnutrition, high-risk pregnancy, and addictions-the byproducts of disparities in social progress. The rate of chronic conditions was similar to that of the general population of Mexico. 